\title{
Channel formation in draining suspensions
}

\author{
Jeanne Bernard and Louison Thorens ${ }^{*}$ \\ Université de Lyon, Ens de Lyon, Université Claude Bernard, Département de Physique, 69342 Lyon, France
}

Received: 17 January 2018 / Accepted: 4 July 2018

\begin{abstract}
We analyse how particles adhering to a wall can create tree-like structures (or flat trees) by an erosive flow. We vary the physical and chemical properties of the fluid and highlight their influences on the patterns observed. We analyse the structures created and conclude that the mass concentration in particles have a clear impact. Using the fractal dimension defined by the box-counting process, we prove that the tree-like patterns are fractal objects. An interesting fact is that this fractal dimension remains constant at a fixed height of analysis.
\end{abstract}

Keywords: Sedimentation / Pattern formation / Fractal

\section{Introduction}

\subsection{Pattern formation in fluids}

The formation of organized patterns in a fluid can often be observed in the day-to-day life and stems from a range of physical phenomena. Among those, we can mention the Marangoni effect [1] (two fluids with diff0erent surface tensions creating a surface tension gradient, seen on wine glasses), the Saffman-Taylor effect [2] (the less viscous fluid producing viscous fingering when penetrating a more viscous one) and the Rayleigh-Taylor instability [3] (two fluids of different densities, creating beautiful vortices, the instability appears when the lighter fluid pushes via an external force the heavier). Other patterns such as those shown in Figure 1 are due to erosion and deposition processes [4]. These types of structures are created when a fluid carrying along solid granular particles is flowing down a wall. Examples of these structures are fractal river basins [5], erosion of volcanic ash, shorelines or even the patterns left on a glass by curdled milk.

We focus in the present article on this specific phenomenon of particle sedimentation in an erosive flow.

\subsection{Suspension vs. colloids}

Such flat trees as shown in Figure 1 are created as soon as water mixed up with solid particles of the appropriate size (for instance aluminium oxide powder [4]) flows down an inclined or vertical hydrophilic surface (the role of the surface will be discussed later). For the flat-tree structures

\footnotetext{
* e-mail: louison.thorens@ens-lyon.fr
}

to appear, the size of the particles is essential: colloids, by definition, do not sediment and therefore cannot create such patterns, whereas bigger particles in suspension can. A colloid cannot sediment because the thermal energy is sufficient to balance its weight. Therefore, a simple calculation consists of comparing this thermal energy with the potential energy:

$$
\frac{1}{2} k_{B} T=\frac{4}{3} \pi R^{3} \rho g h
$$

One can consider that the particle does not sediment if its displacement is of the order of its diameter, therefore our criterion is the following: $h=2 R_{\min }$. Finally, we show that the minimal radius of particles is given by:

$$
R_{\min }=\sqrt[4]{\frac{3}{16} \frac{k_{B} T}{g} \frac{1}{\pi \Delta \rho}}
$$

where $k_{B}$ is the Boltzmann constant, $T$ the temperature, $g$ the gravitational acceleration and $\Delta \rho$ corresponds to the difference between the density of the particles and the density of the fluid. The particles size is chosen according to equation (2), in our case we find $R_{\min }=0.6 \mu \mathrm{m}$. To verify experimentally this quantity, we took a powder of $1 \mu \mathrm{m}$ diameter particles, and did not notice the flat-tree phenomenon.

The determination of a maximal size of the particles allowing the formation of flat-tree patterns is more delicate. This limit depends not only on the thickness of the fluid film on the wall $(50 \mu \mathrm{m})$ but also on the interactions between the particles and the surface, which are notoriously difficult to fully describe and quantify. 


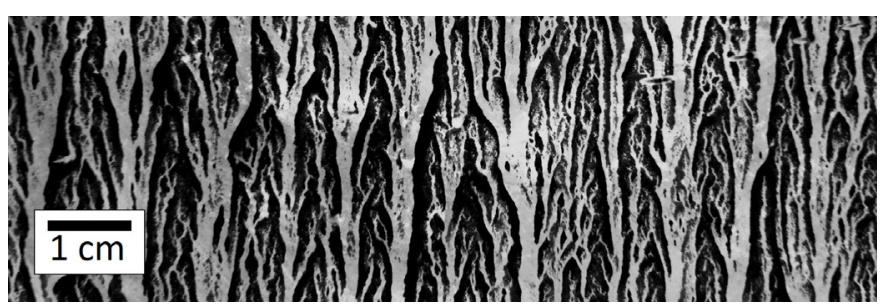

(a)

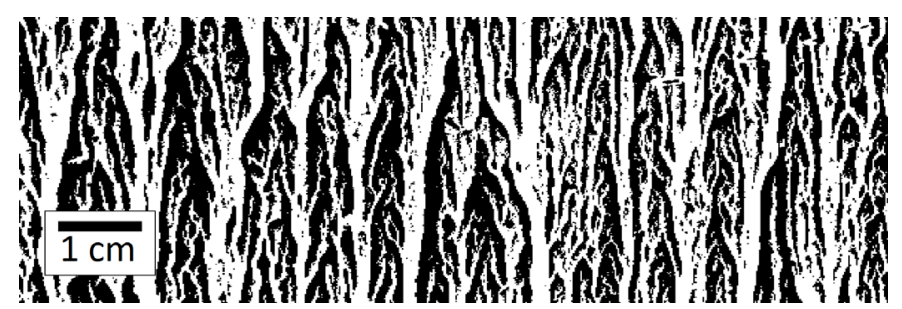

(b)

Fig. 1. Typical example of a flat tree created using a mixture of distilled water and commercial abrasive powder of cerium oxide 1a, and its binarized version $1 \mathrm{~b}$.

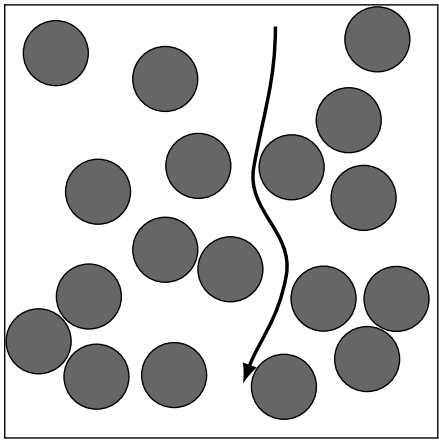

(a)

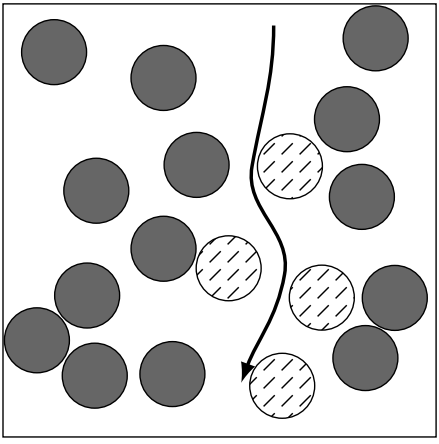

(b)

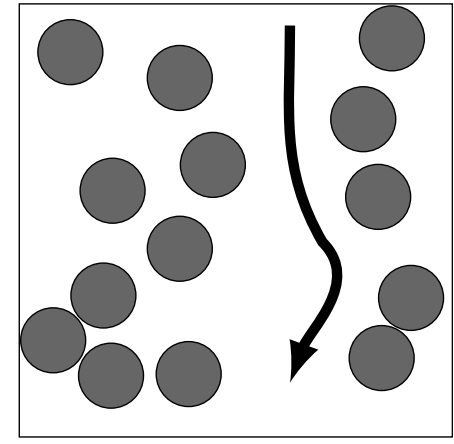

(c)

Fig. 2. Presentation of the pattern formation mechanism. The liquid flows down the wall (represented by the arrow in $2 \mathrm{a}$ ), mostly in the regions where the constraint due to the particles (sticking to the wall) is weaker. Since the fluid mostly flows in these regions, some particles are detached from the wall (shaded in $2 \mathrm{~b}$ ), the concentration decreases and the constraint too. Therefore the flow increases in these regions (thicker arrow in 2c) and the phenomenon is amplified.

\section{Method}

\subsection{Theoretical modeling}

Creating tree-like structures only requires a fluid (for instance distilled water), solid particles (in our experiments cerium oxide abrasive powder) and an inclined hydrophilic surface. The experiment can either start with mixing up the fluid and the particles, or with the particles already poured on the surface ${ }^{1}$.

As illustrated in Figure 2, the pattern formation is generated by a loop phenomenon. When the fluid flows down, it encounters some regions of smaller resistance to the flow, and due to initial inhomogeneities in the repartition of the particles in the mixture, some preferential channels begin to appear (Fig. 3). This is exactly like trying to move fast through a crowd: one is sneaking in the

\footnotetext{
$\overline{1}$ In both cases a strong analogy with fractal river basins, liquid yoghurt [7] or honey [8] can be made.
}

less dense areas. Meanwhile, particles stick to the wall because of electrostatic forces (van der Waals interactions), as illustrated in Figure 4. A balance at removal gives us the following relation:

$$
\begin{gathered}
\left\|\vec{F}_{\text {van der Waals }}\right\|=\left\|\vec{F}_{\text {weight }}\right\|+\left\|\vec{F}_{\text {fluid }}\right\|, \\
\frac{A R}{12 D^{2}}=\frac{4}{3} \pi R^{3} \rho g+6 \pi \eta R v,
\end{gathered}
$$

where $A$ is the Hamaker constant, $R$ the radius of a particle, $D$ the mean distance between the wall and the particle, $\rho$ the density of a particle, $\eta$ the viscosity of the fluid and $v$ its local velocity. From there, the fluid carries away the remaining particles that are located in low density areas, do not stick to the surface and are unable to block or deviate the downward flow. Therefore, tree-like patterns develop in a loop process. This model is confirmed by numeric simulations of sedimentation process as presented in [9]. 


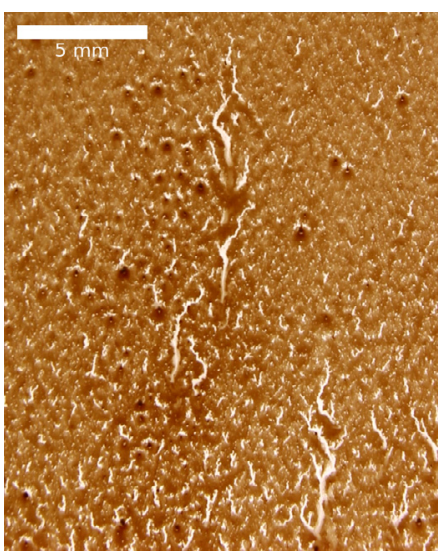

(a)

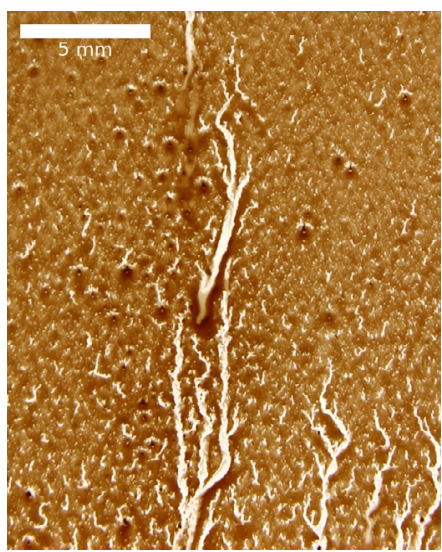

(b)

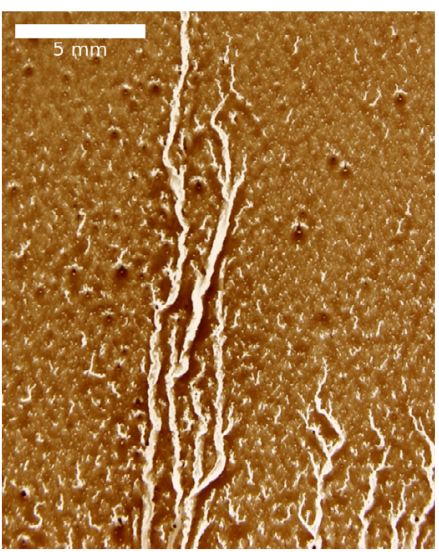

(c)

Fig. 3. Screenshots of the pattern formation. The inhomogeneities in 3 a create channels in $3 b$ that are amplified in $3 c$. These snapshots confirm the process illustrated in Figure 2. The full video can be seen in [6].

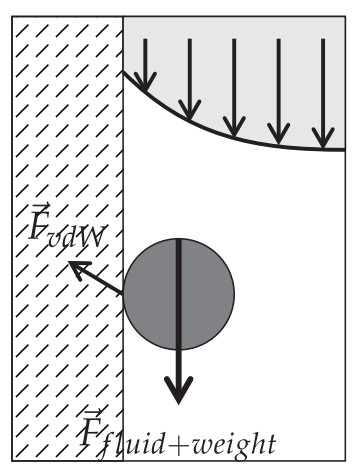

Fig. 4. Cross section of our experiment. The particle sticks to the wall by van der Waals interactions, and the fluid applies a force $\vec{F}$ on the particle. The particle is carried away by the fluid when the van der Waals interactions are too weak to compensate the weight of the particle and the fluid constraint.

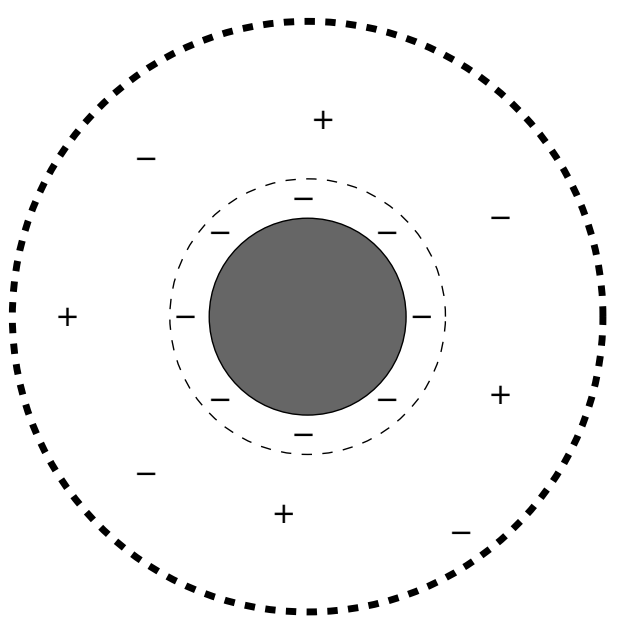

Fig. 5. Theoretical view of a partical surrounded by its negatively charged barrier in a salted medium. The cation of the salt screens the barrier, revealing the short distance interaction.
This formation of flat trees is very similar to erosion processes: the fluid flow removes some particles, and just like sediments, other particles are transported from one place to another, or removed from the wall. A strong analogy with the formation of river deltas can be made: they are almost entirely made of sediment eroded from the banks and bed of rivers.

Particularly striking and beautiful is the fact that the regular and fractal nature of the flat trees still remains a mystery. We understand how the structures are formed, but do not know why they are so regular and reproducible.

\subsection{Influence of $\mathrm{pH}$ and salinity}

Finally, we want to present briefly the theoretical influence of $\mathrm{pH}$ and salinity on the formation of our patterns.

The Hamaker constant depends on the salinity and $\mathrm{pH}$ of the medium [10]. By lowering the $\mathrm{pH}$ or increasing the salinity we reveal the short range interaction of the particles. The explanation is the following: the particles are negatively charged on surface, by adding an acid (decreasing the $\mathrm{pH}$ ) we introduce in the medium some positively charged ions $\mathrm{H}_{3} \mathrm{O}^{+}$. Therefore, the negative barrier around the particles is screened, and the particles can now interact at a shorter distance. This is the same phenomenon than when we add salt composed of anions $\left(\mathrm{Cl}^{-}\right)$and cations $\left(\mathrm{Na}^{+}\right)$. A quick representation is given in Figure 5.

\subsection{Details and protocol}

Throughout our study, we use a cerium oxide commercial abrasive powder, chosen according to the literature [4] and for practical reasons. Its density being $7.215 \mathrm{~g} \mathrm{~cm}^{-3}$, equation (2) gives, at room temperature, a minimal radius of $10^{-7}$ for the particles mixed up with distilled water. Therefore, we chose an abrasive powder of $3.5 \mu \mathrm{m}$, an order of magnitude above the theoretical limit.

In order to make reproducible experiments, several precautions were taken. First of all we used always the same abrasive powder in our experiments: fine grade 


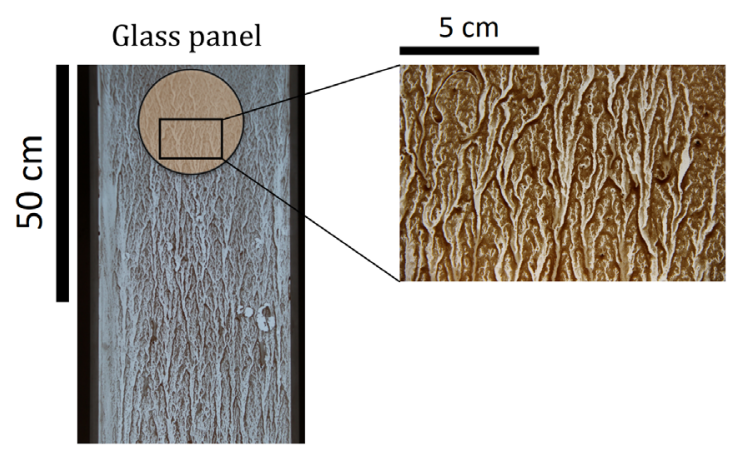

Fig. 6. Choice of the constant height of analysis. The orange disk represents the position and size of the crystallizer we used for all our experiments. The following study is made at constant height in order to allow reproducible and comparable results.

cerium oxide, a polishing solution for glass with an average particle size of $3.5 \mu \mathrm{m}$. These particles are chemically inactive in water and their size is well adapted to create flat trees. The powder is mixed up with distilled water in controlled proportions using a precision balance, and the use of a $20 \mu \mathrm{m}$ sieve minimizes the formation of aggregates. The proper mixture is then realized with strong agitation combined with the use of an ultrasonic homogenizer, allowing a uniform distribution of the particles in the fluid.

We create flat trees by pouring the mixture in a flat glass container (in our case a $20 \mathrm{~cm}$ large crystallizer) which generates a vertical or inclined flow. The main properties required regarding the surface used should be hydrophilic (otherwise no patterns are observed due to the lack of interactions between the fluid and the wall) and transparent (to enable us to take pictures with high contrast and sharpness using a homogeneous light source behind the glass container). Firstly, a thin layer of fluid covers the surface within a few seconds. Then this layer destabilizes and the erosion patterns appear, on a longer time-scale of about $100 \mathrm{~s}$.

Since the growing of the tree-like structures does not stop as long as the wall is long enough, a choice had to be made when analysing the patterns. This choice is shown in Figure 6: our whole study is conducted at a fixed height corresponding to an $8 \times 12 \mathrm{~cm}$ region located after the fluid has flowed along a distance of $10 \mathrm{~cm}$. This choice is totally arbitrary but was strictly respected all along our study. We preferred to study the impact of the physical and chemical properties on the shape of the patterns, and therefore the impact of height (as shown in Fig. 6 with the glass panel) is not studied in this paper.

\section{Results}

\subsection{Mass concentration}

Regarding the influence of the mass concentration of the particles in the fluid, our results shown in Figure 7 indicate that below a mass concentration of $20 \%$ for the particles, it is difficult for the bare eye to distinguish a repeated pattern. Computational analysis is necessary for further quantitative conclusions (see next section).

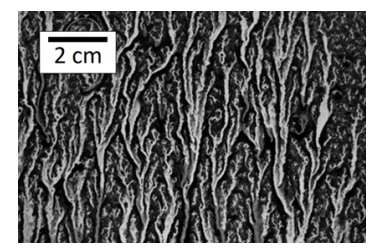

(a) mass concentration: $42 \%$

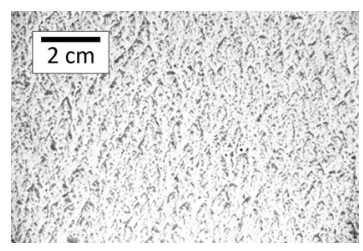

(b) mass concentration: $15 \%$
Fig. 7. Varying the mass concentration of the cerium oxide particles in distilled water clearly changes the shape of the flat trees.

\subsection{Image processing}

The first step of each process is to binarize the picture as follows:

- 0 (black) for powder;

-1 (white) for water.

To do so, we compare the value of each pixel to the average value in the whole picture. This binarization is arbitrary, but after a few tests by changing the threshold, we did not see any strong difference in our results. The error on the binarization is taken into account as error bars in our plots.

Instead of using a classical fast Fourier transform, and in order to fully analyse the patterns, we propose to use the auto-correlation function given by the following equation:

$$
R_{p}(x, y)=\int_{-\infty}^{+\infty} p(x+\delta, y) p(\delta, y) \mathrm{d} \delta,
$$

with $x$ and $y$ respectively representing the horizontal and vertical coordinate.

The idea here is to compare the picture to an exact copy of itself shifted in the horizontal direction. Assuming that the patterns follow a privileged direction (vertical), and that these patterns do not change significantly over the studied window, we perform our analysis on each row of the picture and then take their average along the $y$ direction.

The typical auto-correlation function for a given massconcentration in shown in Figure 8. By definition of the function, the resulting graph is symmetrical with respect to the vertical axis, with the normalized maximum appearing at the origin. On one hand, the minimum of correlation corresponds to the horizontally averaged black-band size (i.e. the powder channel typical size). On the other hand, the $\mathrm{x}$-intercept of the first slope represents the horizontally averaged size of the white lines (i.e. the average water channels typical size). Here, "water channel" means the empty channel left by the flow of water.

To verify that our technique of auto-correlation function is correct, we compare the ratio of average water and powder channels sizes and the ratio of the numbers of white pixels over the number of black ones. The comparison is given in Table 1.

We notice here that the ratios are in good agrement with the errors computed over rows of the images, with a small variation for the lowest mass concentration, certainly due to the high sensibility of the image processing to the 


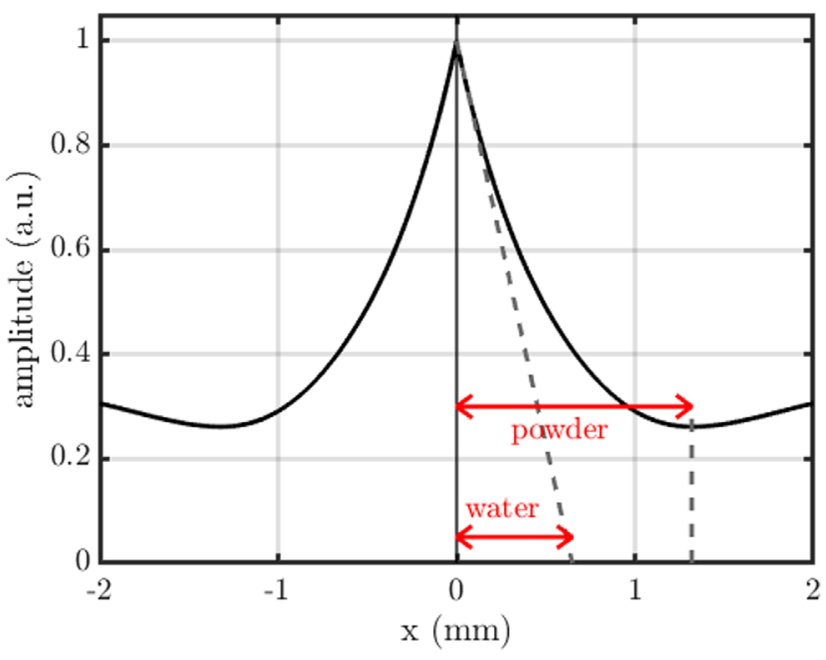

Fig. 8. Auto-correlation function for a given mass concentration. The functions drop to an anti-correlation minimum that corresponds to the powder-channel typical length. The x-intercept of the first slope leads to the water-channel typical length.

Table 1. Comparison table.

\begin{tabular}{lcccccc}
\hline$\varphi_{m}$ & $15 \%$ & $20 \%$ & $25 \%$ & $30 \%$ & $35 \%$ & $42 \%$ \\
\hline $\begin{array}{cccc}\frac{\text { size }}{\text { size }_{\text {powder }}} \\
N_{\text {phite }}\end{array}$ & 1.14 & 1.03 & 0.75 & 0.71 & 0.65 & 0.49 \\
$N_{\text {black }}$ & 1.26 & 1.13 & 0.81 & 0.76 & 0.69 & 0.53 \\
Error & 0.20 & 0.15 & 0.13 & 0.09 & 0.07 & 0.06 \\
\hline
\end{tabular}

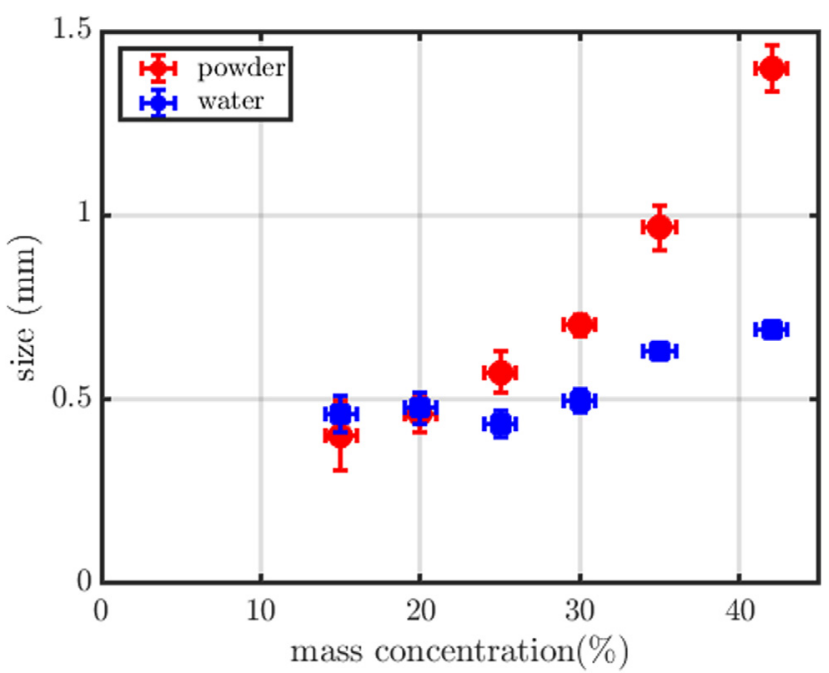

Fig. 9. Sizes of powder and water channels for different mass concentrations found by the auto-correlation method. Both sizes increase with the mass concentration.

binarization at this value (as seen in the error bars in Fig. 9). We notice that we find the same tendancy of the ratios in both cases, with a transition appearing at around $20 \%$. The resulting graph of the different sizes found by the auto-correlation function is given in Figure 9. It is now clear

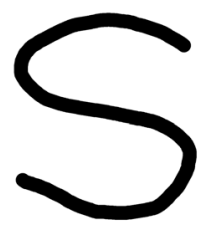

(a)

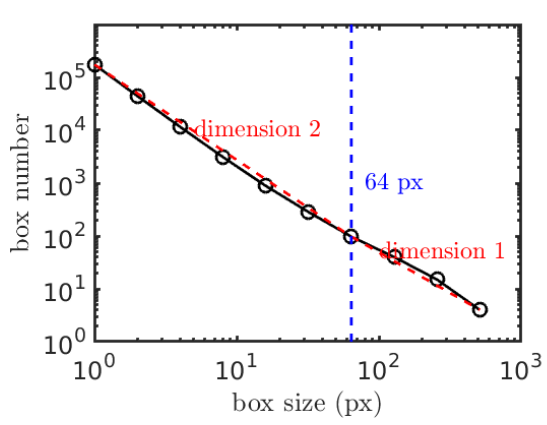

(b)
Fig. 10. Box-counting code result. The slope is between 1 and 2 as expected for a 2D-pattern. The linear tendency is strong for the whole range of box sizes; we can conclude that our patterns are fractal objects.

that the mass concentration of the particles in the fluid has a significant impact on the erosion patterns: both sizes increase with the mass concentration.

Completing our study of these tree-like structures, we focus on the fractal dimension of such complex objects. The fractal dimension has been detailed in previous works [7] for different types of powder. We wanted to show the same behaviour by varying the mass concentration of the suspension. As presented in this section, this fractal dimension analysis highlights the fractal nature of the flat trees. We use here the definition of the fractal dimension based on the box-counting principle. The image is divided in squares of a given size $\varepsilon$ and the number of squares containing black pixels $N(\varepsilon)$ is counted. Decreasing the size of the squares, from the size of the image to one pixel, the fractal dimension is defined as follows:

$$
D_{\text {box }}=\lim _{\varepsilon \rightarrow 0} \frac{\log (N(\varepsilon))}{\log (1 / \varepsilon)} .
$$

Hence, the fractal dimension is given by a number between 1 and 2 for a 2D-pattern.

To measure this fractal dimension, we use the MATLAB code in [11] and plot $\log (N(\varepsilon))$ against $\log (\varepsilon)$, the fractal dimension $D$ being the absolute value of the slope in the resulting plot. From Figure 10, one can see the strong fractal behaviour of our patterns.

We give two basic examples of fractal analysis by boxcounting in Figures 10-12.

The resulting fractal dimension for different mass concentrations is plotted in Figure 13. These results clearly reveal not only that the flat trees are fractal objects, but also that their fractal dimension is actually independent of the mass concentration of the particles in the fluid. The fractal dimension value of $D \approx 1.8$ which is confirmed by [7], where yoghurt, talcum powder and flour suspensions all form tree-like structures with a fractal dimension of $D \approx 1.6-1.75$.

Finally, we did not discuss in the present article the impact of the wall inclination. We did not notice any clear 


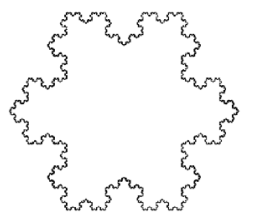

(a)

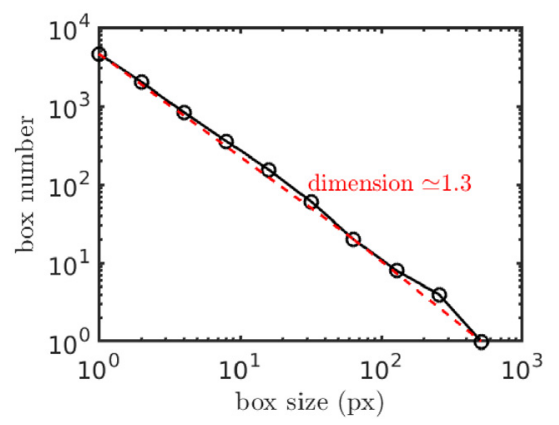

(b)
Fig. 11. Example of boxcounting fractal analysis. We analyse here the fractal dimension of a $64 \mathrm{px}$ thick line, on the boxcounting analysis we clearly see the transition of fractal dimension from 1 (line) to 2.

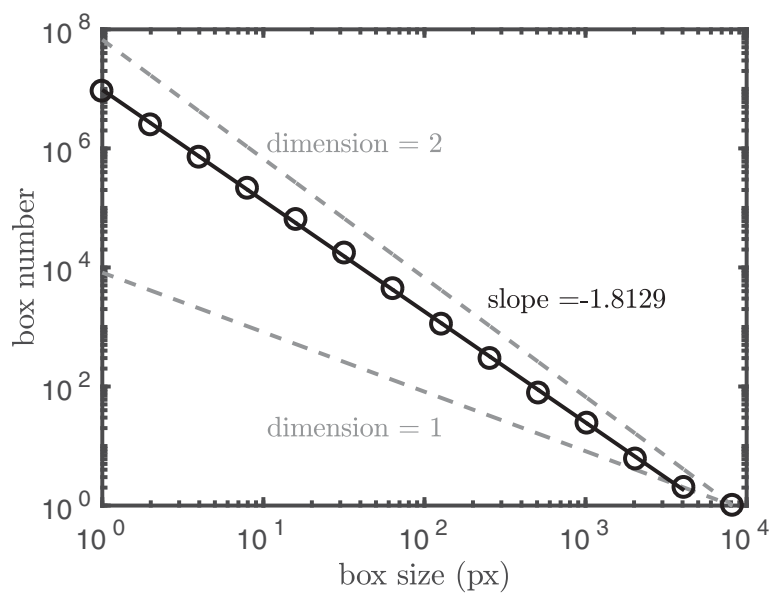

Fig. 12. Example of boxcounting fractal analysis. We analyse here the fractal dimension of a Koch snowflake, the fractal dimension is known to be of 1.26 , in good agreement with our measurement limited by the image definition.

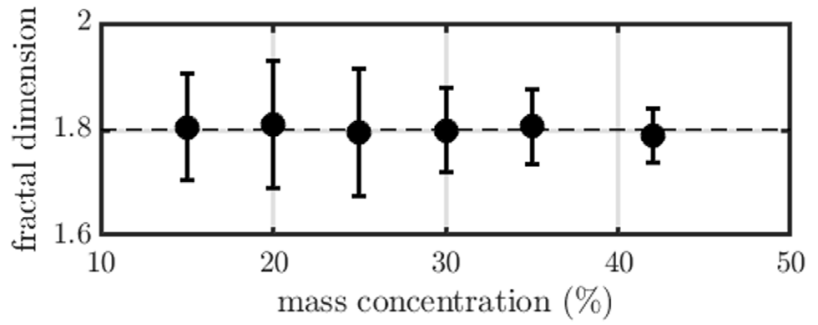

Fig. 13. Fractal dimension for different mass concentrations and viscosities. It is clear that the fractal dimension is shown to remain constant when the mass concentration is modified. Here, the errobars correspond to the standard deviation of the fractal dimension according to the different thresholds of image binarization.

change in the tree-like patterns when the surface was more or less tilted. It appears that the wall inclination only changes the patterns formation dynamics: the smaller the inclination, the slower the patterns formation.

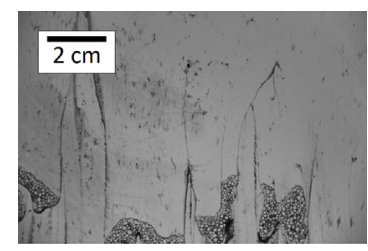

(a) Distilled water and a few drops of SDS, the surface tension of the fluid is decreased.

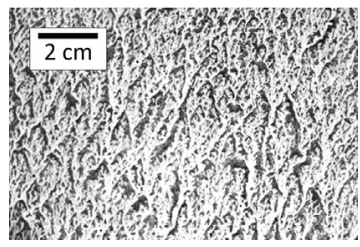

(c) Distilled water with salt.

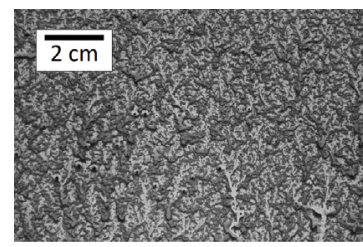

(b) Distilled water with a few drops of hydrochloric acid: $\mathrm{pH}=1$.

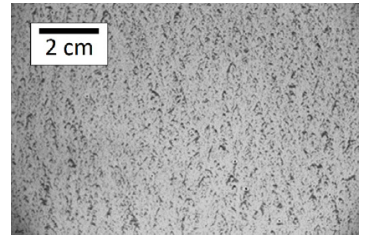

(d) Distilled water and glycerol: viscosity increased 17 times.
Fig. 14. Changing the $\mathrm{pH}$ or adding some salt modifies the chemical interactions between particles. The mass concentration is here constant at $30 \%$.

\section{Discussion}

On the use of the auto-correlation function, our results are in good agrement with the verification of the white vs. black pixels ratio, the small discrepencies between both ratios can be explained by the threshold of binarization but also on the vertical approximation we make when we compute the auto-correlation function. All these considerations lead us to the conclusion that our results on the analysis of mass concentration are correct.

Furthermore, we analysed the fractal dimension of our patterns. As expected, we found a constant D even though it is slightly higher than the values observed in the litterature. This discrepency can be explained by the use of a different powder size (smaller in our study), maybe surface interactions or any other experimental discrepancies. Nevertheless, the $\mathrm{pH}$ does not seem to have an influence, since we fond a fractal dimension of about 1.78 when our solution has $\mathrm{pH}=1$.

\section{Dead end}

Regarding the physical phenomenon responsible for such beautiful patterns, we unfortunately do not fully understand the reproducibility of the flat trees and their fractal nature. The model presented in the method section is deduced from the experiments: it remains very simple, providing only a basic understanding of the phenomenon.

We tried to use surfactant such as dishwashing liquid and sodium dodecyl sulfate (SDS). The impact of both can be seen in Figure 14. The idea here was to vary the surface tension of the liquid to see the impact on the formation of flat trees. Although the impact seems to be significant as we can see on the pictures, the formation of bubbles and 
foam in the suspension prevents a clear study of the phenomenon. The main restriction is on the preparation of the solution: because we need to shake a lot the suspension (we recall that the powder sediments) before spreading it on the surface, it is almost impossible to prevent the formation of foam. This constraint is clearly seen with the use of SDS.

Finally, we explained in this paper the theoretical influence of $\mathrm{pH}$ and salinity on the patterns. Figure 14 gives an overview of both properties of the fluid. Unfortunately, we did not succeed into the analysis of such patterns. In fact, the vertical approximation made for the use of auto-correlation function does not stand anymore for such patterns. Here, the phenomenon is both sedimentaiton and jamming process as detailed in [8]. It is the same phenomenon which occurs when the viscosity increases (as in honey for example). A more in-depth study is needed to fully apprehend the impact of $\mathrm{pH}$ or salinity, we decided to focus our analysis on mass concentration in this paper. Nevertheless, by increasing the viscosity, the Stokes force acting on the particles increases. More particles are carried away by the flow, and the trees are drastically erased; and lowering the superficial tension implies that the fluid offers less resistance when it encounters aggregates, and less particles are carried away by the flow.

\section{Conclusion}

The simple properties required to form flat trees are a fluid and particles in suspension in it, allowing erosion patterns to be formed on a surface. In this study, we investigated a key parameter affecting the shape of the observed structures: mass concentration of particles in the fluid.

Quantitative tools, such as the auto-correlation function and the fractal dimension, are used to analyse these patterns.

Our results - obtained at fixed height of analysis show that the characteristic size of the channels left by water or the powder channels increases when the initial concentration of particles in the fluid increases. We also demonstrated that such flat trees are fractal objects. Interesting is the fact that their fractal dimension does not depend on the mass concentration of particles. This fractal dimension is approximately equal to 1.8 , which is consistent with the fact that these patterns are twodimensional.

To go further in our study of the flat trees we plan to investigate the effect of the temperature. We expect the temperature to modify the minimum radius required for the particles to create tree-like patterns. Moreover, we plan to investigate further the role of the $\mathrm{pH}$, which is a more delicate study since it affects both the interaction of the particles with the wall and the interaction between particles.

This study was conducted as part of our research to prepare the 9th International Physicists' Tournament (http://2017.iptnet. info/), which proposed the following problem: "Some liquids do not flow freely down the walls of a vessel, but form tree-like structures. Which properties are required for liquids to behave in this way? Explain how these properties affect the shape and characteristic dimensions of the structures that are formed by these liquids."

We would like to thank Nicolas Taberlet and Nicolas Plihon not only for their help when we conducted the experiments, but also for the comments on the manuscript. We would like to thank also Jérémy Ferrand for his help, Tristan Guyomar for the useful discussions, and Yannick Bertrand for his precious help during revision of this paper. A special thanks to Adrian Daerr, author of [4], for the discussion we had at the French Physicists' Tournament.

\section{References}

1. J. Thomson, On certain curious motions observable at the surfaces of wine and other alcoholic liquors, Philos. Mag. 10, 330-333 (1855)

2. P.G. Saffman, G. Taylor, The penetration of a fluid into a porous medium or hele-shaw cell containing a more viscous liquid, Proc. R. Soc. A: Math. Phys. Eng. Sci. 245, 312-329 (1958)

3. L. Rayleigh. Investigation of the character of the equilibrium of an incompressible heavy fluid of variable density, Proc. Lond. Math. Soc. s1-14, 170-177 (1882)

4. A. Daerr, P. Lee, J. Lanuza, É. Clément, Erosion patterns in a sediment layer, Phys. Rev. E 67, 1-2 (2003)

5. M. Cieplak, A. Giacometti, A. Maritan, A. Rinaldo, I. Rodriguez-Iturbe, J.R. Banavar, J. Stat. Phys. 91, 1-15 (1998)

6. L. Thorens. Formation of "flat trees", https://youtu.be/ ZN0k67KMGlk.

7. N. Maleki-Jirsaraei, B. Ghane-Motlagh, S. Baradaran, E. Shekarian, S. Rouhani, Fractal behaviour of flow of inhomogeneous fluids over smooth inclined surfaces, J. Phys. Condens. Matter 16, 2497-2505 (2004)

8. M. Buchanan, D. Molenaar, S. de Villiers, R.M.L. Evans, Pattern formation in draining thin film suspensions. Langmuir 23, 3732-3736 (2007)

9. M.R. Errera, A. Bejan, Deterministic tree networks for river drainage basins, Fractals 06, 245-261 (1998)

10. R.M. Pashley, M.E. Karaman, Van der waals forces and colloid stability, in: Applied Colloid and Surface Chemistry, John Wiley Sons Ltd, 2005, pp. 127-151

11. F. Moisy, Fractal dimension using the 'box-counting' method for 1D, 2D and 3D sets, MATBAL Central File Exchange. Retrieved June 10, 2008.

Cite this article as: J. Bernard, Louison Thorens, Channel formation in draining suspensions, Emergent Scientist 2, 4 (2018) 